\title{
Pregnancy and Heart Transplantation. First Case in Mexico
}

\author{
Hugo Jesus Zetina-Tun ${ }^{1}$, Raul Pedraza Grijalva ${ }^{2}$, Guillermo Careaga-Reyna ${ }^{3^{*}}$, Carlos Lezama-Urtecho ${ }^{4}$ and \\ Jose Galvan-Diaz ${ }^{5}$ \\ ${ }^{1}$ Coordinator Thoracic Organ Transplant Clinic. UMAE Hosp General "Gaudencio González Garza" CMR IMSS, Mexico \\ ${ }_{2}^{2}$ Intensivist, Thoracic organ transplant clinic. UMAE Hosp General "Gaudencio González Garza" CMR IMSS, Mexico
}

${ }^{3}$ General Director and Chief Surgeon of Thoracic Organ Transplant Program. UMAE Hosp General "Gaudencio González Garza" CMR IMSS, Mexico

${ }^{4}$ Chief of Cardiothoracic Surgery Division. UMAE Hosp General "Gaudencio González Garza" CMR IMSS, Mexico

${ }^{5}$ Cardiologist. Department of Cardiothoracic Surgery De la UMAE Hosp General “Gaudencio González Garza” CMR IMSS, Mexico

Received: April 29, 2018; Accepted: June 05, 2018; Published: June 12, 2018

*Corresponding author: Guillermo Careaga-Reyna, General Director and Chief Surgeon of Thoracic Organ Transplant Program. UMAE Hosp General "Gaudencio González Garza" CMR IMSS, Mexico, E-mail: gcareaga3@gmail.com

\section{Abstract}

The success of cardiac transplantation has included female recipients of childbearing age. Pregnancy is risky in these patients because of possibility of acute rejection and lost graft, birth defects of the product and complications related to pregnancy.

Case report: 35 years old female at time heart transplant 26 months, before she present with pregnancy unscheduled. During the same we switched to azathioprine and eliminated mycophenolate mofetil; tacrolimus was continued as immunosuppressive therapyShe was monitored every 3 months with tacrolimus levels, echocardiography and last two months of pregnancy every 3 weeks, narrow prenatal control. Gestation had a normal evolution, no complications in heart graft, diseases related to pregnancy. Pregnancy finished by cesarean section and a male live product was obtained without congenital malformations. Control graft 3 months post cesarean with LVEF $60 \%$.

Conclusion: Pregnancy in recipients of heart transplant can be completed successfully, it is best to suggest prenatal care, and no pregnancy. If recipient want it has to be programmed and one year after cardiac transplantation without periods of severe rejection.

Key words: Heart Transplant; Pregnancy; Acute Rejection; Immunosuppression;

\section{Introduction}

Cardiac transplantation (CT), it has been considered the ideal treatment for terminal cardiac failure. With the time and experience acquired, the recipients have been younger and women of childbearing age coming to have pregnancies with the involved risks [1].

Pregnancy after cardiac transplantation is possible but is associated with an increase in the risk of obstetric complications, including pre-eclampsia, delay in the rising fetal treatment and pre term births [2]. The first successful pregnancy in a CT recipient occurred in 1986 and was reported at 1988 by Lowenstein. Since then the reports of isolated cases and small series has been more and more frequently published [3]. Most of these pregnancies have favorable results, although less than the observed in normal pregnancies. However, this group of patients remains a challenge for physicians dealing with them [3].

Pregnancy is a complex condition, and more in women transplanted of heart. On this area there are controversies like the following related to the mother: Risks to her short and long term health care, for the allograft: Risk of dysfunction or loss of the graft, use of drugs, the metabolism changes that could increase their susceptibility allograft rejection and in relation to the fetus: teratogenic effects of the immunosuppressive drugs [4].

Since the introduction new immunosuppression therapies, rejection rates in the first year after transplantation have decreased considerably [2-11]. On this basis, pregnancy can be safe after the second year of the transplant. Its applied to the recipients of heart and kidney transplant, but ideally it is recommended to have a contraceptive or non-pregnancy control. This recommendation is based on a large extent in the risk of rejection.

According to the American Transplant Society in the consensus conference on reproductive questions, individual factors should be considered when offered planned pregnancy opportunities, but $50 \%$ of the pregnancies are not programmed $[5,10]$.

The issues addressed at this meeting were: Risk of acute rejection and infection, and pharmacological treatment with potential risk effects as toxic, teratogenic and graft function.

We present the first case In our country of a heart transplant patient who had a pregnancy which was successfully resolved.

\section{Clinical case}

A 35 years-old female with dilated cardiomyopathy and AV blockade, received an ortothopic bicaval heart transplant on 
January $16^{\text {th }}, 2013$ in our hospital.The post transplant evolution has been favorable in functional class I of the New York Heart Association (NYHA). The Initial immunosuppressive therapy was employed: prednisone, mycophenolate mophetil and tacrolimus. The endomyocardial biopsy (EMB) showed as maximum score 1-R of 2004 ISHLT classification [5]. The prednisone was suspended at 11 months post-transplant and its control EMB with cell rejection 1-R (ISHLT). Other prescribed medications: omeprazole, pravastatin, acetylsalicylic acid and propafenone (This due to the presence of supraventricular extrasystoles since the 6 months post-transplant and EMB without data of histopathological cellular or humoral rejection. In the 12th month, deep venous insufficiency of the right leg is detected. The last EMB on February 2014 was without rejection and was confirmed the absence of coronary obstructive lesions too.

The obstetrics data G: II, P: I, A:0. The date of last pregnancy in 2003. The contraceptive control of the patient was pharmacological for eight years, and later with the rhythm method, with which it continued after transplantation, as other hormonal methods were not indicated by the thromboembolic risk and the intrauterine device was nor used by the risk of infection.

In March 2015 with amenorrhea an ultrasound confirmed 8-weeks intrauterine pregnancy. The continuity of pregnancy was determined by the patient listening to the opinions of the medical group in charge of his post-transplant care, supported by obstetrics specialists and literary reports, which indicated the successes of recipient women transplanted from heart or kidney. So, with obstetrician follow-up, a control program of the cardiac graft and the following maternal-fetal binomial which consisted in avoid the use of propafenone, acetylsalicylic acid and pravastatin, immunosuppressive scheme modification: Remove mophetil micophenolate and start azathioprine $50 \mathrm{mg} /$ day, take serum tacrolimus levels every three months until $7^{\text {th }}$ month of gestation and monthly for the last two months, an echocardiogram at rest: Initial, every three months, and monthly in the last two months of gestation. Perinatal obstetric control: every two months and last month every 15 days, the patient received folic acid, B-Complex and iron supplements. Azathioprine dose was calculated in order to maintain the total leukocytes between 3.500 and $6.000 / \mathrm{mm} 3$.

The first echocardiogram showed normal function of cardiac allograft with left ventricle ejection fraction(LVEF) 65\% and pulmonary artery systolic pressure (PASP): $35 \mathrm{~mm} \mathrm{Hg}$. There was no decrease in LVEF, nor increase on PASP. Control within two months prior to the pregnancy term with left ventricle diastolic diameter(LVDD): $35 \mathrm{~mm}$, LVEF 60\%, and PASP: $30 \mathrm{~mm} \mathrm{Hg}$. Without pericardial effusion. Initial tacrolimus levels were 5.73 $\mathrm{ng} / \mathrm{mL}$.

During gestation there were no complications attributed to cardiac allograft, or other related to gestation.

An elective cesarean operation was employed to conclude pregnancy in November 5, 2015, with obtaining unique live product, male sex, with weight of $3150 \mathrm{gr}$ and an Apgar score of 9.9, without apparent congenital malformations. In the surgical procedure, we proceeded to perform a contraceptive method by bilateral tubarian occlusion. Patient was delivered from hospital at $7^{\text {th }}$ postsurgical day after caesarean section. It was recommended to avoid breast feeding the newborn by risk of immunossupresive drugs excretion of drugs in breast milk and the product feeds on formula milk. After delivery the mophetil micophenolate was reintroduced to the patient.

The 40 days post-delivery patient was in NYHA functional class I, with tacrolimus levels of $12.5 \mathrm{ng}$ and echocardiogram with LVEF $60 \%$. New control of tacrolimus at 90 days with levels of: 12 ng/ML and echocardiogram with LVEF: 62\%.

The four-month control biopsy was without acute rejection, the tacrolimus levels in therapeutic range $8 \mathrm{ng} / \mathrm{mL}$. The echocardiogram with a LVEF 70\%. Right cardiac catheterization with right pulmonary artery pressures in normal ranges of $32 / 10$ $\mathrm{mm} \mathrm{Hg}$.

The endomyocardial biopsies and right cardiac catheterization was made through venous approach

At 24 months after the childbirth, the graft is with normal function, the LVEF: 65\%. The biopsy EMB without rejection evidence and the total dose of tacrolimus by levels above $10 \mathrm{ng} /$ Ml. In the follow-up there were no infectious complications. The male product with normal growth and development.

\section{Discussion}

The increase in pregnancies in women of childbearing age, recipients of transplant of solid organs, has been more frequent, initially in those with renal transplantation in 1958 [5], later women with other types of solid organ transplantation have been achieved. In 1986, the first successful pregnancy was documented in a woman with heart transplant. Each time the number of women of child bearing age are benefited from a heart transplant and require extreme care during and after gestation.

Without a doubt the best option for a patient transplanted heart in reproductive age, is to avoid pregnancy. However, the possibility that the patient in a scheduled or unplanned way becomes pregnant exists and is a challenge for those who are in charge of the care of the case.

The women of childbearing age with solid organ transplantation require extreme care during and after gestation. This is because the gonadal-hypothalamic dysfunction and recovery of the infertility pretransplant within the first few months of the solid organ post-transplant [12] and the chances of pregnancy increase.

Unfortunately, between 50 to $82 \%$ of pregnancies are unscheduled, as happened with our recipient, who did not have regular contraception control [11-13].

On this scenario, it was necessary for the expert groups define care conditions to achieve the proper outcome of pregnancies that occur in this group of patients.

The AST consensus conference on reproductive issues and transplantation [4] sets the following recommendations to heart transplant recipients who wish to get pregnant on an elective: Absence of rejection over the past year functioning and stable graft, which does not exist evidence of acute infections that may affect the fetus, which can stable of immunosuppressants, 
the age maternal, presence or not of comorbidities that may affect the pregnant and the function of the graft and the need for strict medical treatment follow, in such a way that there is no contraindication for the continuity of pregnancy, with the consent of the patient and the joint assessment by a multidisciplinary group that includes perinatology, obstetrics and of course the heart failure team. The decision to interrupt the pregnancy depends on the time of transplantation, the history of frequency and magnitude of rejection histological in the biopsies, with hemodynamic impact; given the above, in this case there was no indication for the interruption of the same and the patient was not raised.

The recommendations for transplanted women of childbearing age are those of carrying out a good control of family planning and, if in the future they want to become pregnant, it should be with programming and under a context of mutual agreement between recipient and medical group with a clear knowledge of possible risks involving immunossupresive therapy in the fetus.

Initial evaluation of a heart transplant recipient who wants to become in pregnant program we must perform the following studies: electrocardiogram/echocardiogram, right catheterization with EMB and potentially coronarography, serum immunosuppressive levels. The hepatic function needs to be assessed and if the patient has some degree of renal dysfunction should be done creatinine depuration of 24-hour and renal ultrasound. Previous results will be useful to comparison with actual renal and hepatic function. Must be carried out blood pressure and urine cultures and the glucose levels studies in the high risk of developing gestational diabetes or preeclampsia [9].

Update the state HIV serological, hepatitis by virus B and C, cytomegalovirus, herpes virus, syphilis, rubella, chickenpox and toxoplasmosis [9].

Blood levels of immunosuppressants should be monitored during pregnancy on the basis of there are physiological changes in the plasma volume, motility and digestive tract absorption, metabolism that can alter the plasma levels of the immunosuppressive drugs [10]. It is very important to have a close surveillanc erelated to rejection during all pregnancy [10].

In normal pregnancy there are systemic cardiovascular changes such: an increase in cardiac output (45\%), heart rate and oxygen consumption (15 to $20 \%$ ) and decrease in the systemic vascular resistance and pulmonary vascular resistance (30\%) [6].

The maximum peak of cardiovascular stress occurs during the labor and birth, specially in the immediate postpartum when heart rate can be $80 \%$ above prepartum value. Heart transplant recipients may have hyperdynamic cardiovascular response during this vulnerable period [6].

A characteristic of the transplanted heart is denervation. The answer to stressis [1] by two physiological adaptative mechanisms: the increased systolic volume in response to increased central venous pressure and volume; and increased heart rate and contractility in response to the elevation of circulating catecholamines. Gestational hemodynamic changes could expose a risk for a transplanted heart, although these induced hemodynamic changes, are usually not bad tolerated [7].

In the postpartum period most patients maintain an adequate graft function. As previously mentioned, alterations in intravascular volume and glomerular filtration alter plasma levels of immunosuppression, due to this post-partum should be monitored regularly for a month and if necessary adjust the doses [3]. At least one post-partum echocardiogram should be obtained and compared with previous echocardiographic data obtained before and during pregnancy. The rest of the monitoring carried out will take the post-transplant patient on a routine basis [3].

As for rejection, it was suggested that pregnancy is a state of immunosuppression, but every time there is more evidence that the immune system does not diminish its activity. It is generally accepted that the rejection rate is not increased. The levels of immunosuppression may decline by the above factors, and an inadequate reduction of immunosuppressants during pregnancy may increase the risk of rejection [3].

Monitoring of patients with require EMB. The pregnancy rejection rate was reported in $11 \%$ of patients in the NTPR study. They were usually mild rejection episodes without clinical consequences and treatment was steroids and increased doses of cyclosporine. In this same study it was reported some cases in the that the pregnancies were not viable. The authors do not mentioned what some other therapeutic strategy would have been used [10].

The postpartum rejection reaction (within three first months), it's been reported at $6 \%$. Steroids are the therapy if choice although the safety of using antithimocyte therapy as rituximab it's unknown. It has been reported in heart transplant patients in the postpartum period antibodies mediated rejection. On this basis, in patients presenting cardiac rejection data during and after childbirth, the levels of PRA anti-HLA must be measured [3].

Successful maternal and fetal outcomes require proper maintenance of the immunosuppression.

The immunosuppressive therapy employed during pregnancy in heart transplant patients include a combination of corticosteroids, calcineurin inhibitors, antimetabolites and macrolide antibiotics [10].

At least 6 weeks before conception, immunosuppressants should be optimized, eliminating mycophenolate, sirolimus and everolimus [9]. Azathioprine should be used instead of mycophenolate for lesser teratogenic effect [10]. Special attention requires women with the presence of frequent nausea and vomiting, to prevent a proper adherence to treatment. Blood levels of immunosuppressants should be monitored during pregnancy taking into account that there are physiological changes in plasma volume [10].

We cannot fail to mention that, in this type of patients, there is a risk of high blood pressure the most frequent complication(46\%), it can produce a premature delivery and delay in fetal growth, pre-eclampsia can occur in a 10 and up to $18 \%[7,11]$.

The risk factors for preeclampsia development include age, chronic hypertension, renal disease, obesity associated with 
insulin resistance and gestational diabetes. Preeclampsia could be difficult to diagnose in this type of patients, as it can attend previously with hypertension and proteinuria. Calcineurin inhibitors increase the uric acid levels. In case of presenting preeclampsia the handling for this does not vary from the obstetric patient without heart transplant [10]

A $30 \%$ patient who have benefited from a cardiac transplantation present diabetes mellitus during the first year. The incidence of presentation of diabetes mellitus during the pregnancy is low and if presents it will be handled based on the obstetric standards care $[10,11]$.

It is known the risk of venous and pulmonary thromboembolism in pregnant patients, this risk compared to pregnant women healthy increases in the pregnant heart transplant recipients [10].

In pregnant patients with high risk of herpes simplex recurrence antiviral therapy should be considered the third trimester. Those that are not immune to the herpes zoster virus, are advised to avoid contact with infected varicella zoster, but if are exposed the use of immunoglobulins is recommended [10].

As to the form of resolution of pregnancy, all birth by delivery is the recommended method in heart transplant patients [3]. Caesarean birth should only be Indicated by obstetrics. In the NPTR study, $40 \%$ of the births were via caesarean section. During labor, must be monitored with electrocardiogram due to high risk of presenting arrhythmias. Invasive monitoring is generally not necessary [3].

Fluid administration should be cautious during the labor and child birth because it can exists an unpredictable response to vasoactive drugs $[3,10]$.

Immediate after child birth, the cardiac output increases due to a associated autotransfusion secondary to uterine involution, it may produce systolic volume and heart rate fluctuations. The heart transplant patients usually tolerate these hemodynamic changes during labor and postpartum. We must remember that there are changes in the glomerular filtration and all the immunosuppressive toxicity [3]

Epidural blockade reduces pain-induced sympathetic response and may reduce acute fluctuations in blood pressure during labor [3].

Due to immunosuppression, this patients haven high risk of early lose of the products. On this basis is reasonable to start a more strictly perinatal control in the week of gestation 32 [1519].

Malformations structurally occurring in 4 to $5 \%$ of pregnant women which is similar to the population in general, (3-5\%), and no pattern different from malformations have been observed in patients with immunosuppressive conventional $[3,11]$.

Although most newborn infants of heart transplant mothers are healthy and develop normally, subsequent follow-up of the children will be necessary [10].

Breast feeding has typically been avoided in these patients. Cyclosporine and tacrolimus is detected in variable concentrations in babies lactating of mothers that consume immunosuppressants. The NTPR study reported breast feeding 89 mothers to their children without reporting adverse effects $[10,11]$.

Because of the benefits breast feeding brings to infants should be carefully weighed against the possible adverse effects of exposure to maternal immunosuppression. The conclusion of the AST Consensus conference was that breast feeding should not be absolutely contraindicated $[9,10,13]$.

In our case our receiver the CT recipient had an unscheduled, detected when there was already a pregnancy 8 weeks by pregnant test. Within the first steps we did was: suspend the mycophenolate (for its effect teratogenic) and changed to azathioprine as suggested by different authors [10], levels of tacrolimus initial and is monitored to maintain between 7-10 $\mathrm{ng} / \mathrm{ml}$. Due to plasma changes, the patient required daily dose increase for maitain those levels. We carry out continuous echocardiograms in order to detect in a timely manner decreases in the LVEF of the allograft, perhaps what we omit and should emphasize in new cases, the realization of fetal echocardiogram to detect congenital cardiac anomalies, and no author reports having done, according to their reports. The perinatal control was very important, receiving the receptor from the gestation diagnosis, until the end of it, not finding complications related to gestation.

The gestation conclusion in our case it was via caesarean section. It was decided on the basis that it was the first case that we face and this joint decision was made to do not expose to patient at elevated cardiac stress that occurs during labor, most obstetric reports recommend vaginal delivery, and only caesarean section by precise obstetric indication [3].

There were no postpartum complications and we restart the immunosuppression at 24 days post-caesarean section. Her discharge was at 7 days to suspend gestation and his current functional class is I NYHA.

That it is a preliminary report and it will be necessary to make a long-term follow-up of the mother and product.

Pregnancy in cardiac transplant recipients is not contraindicated, it is recommended to be elective, scheduled, to optimize the conditions of the same and guarantee success for the binomial. The guides of the International Society of Heart transplant and Lung (ISLHT), recommended at least one year of post-transplant so that the recipient can become pregnant, but they should be informed about the risks involved.

\section{Conclusion}

Undoubtedly in the era of solid organ transplants, we are facing many challenges. Young recipients in fertile age are increasing, coupled with the need and desires to have off spring, conception and pregnancy and recipient women will present more often. In our case, it is the first pregnancy that occurs in Mexico of a woman receiving cardiac transplant. How is mentioned in medical literature reports, the largest of these pregnancies are unscheduled. In this case the gestation was unexpected, unscheduled, which led us to establish measures aimed at monitoring the mother-child binomial following the 
recommendations of the ISHLT. The management must be individualized according to the clinical condition of the mother and the current state of the cardiac graft $[15,16]$.

The recipient must be in full awareness that pregnancy is a state of high risk for cardiac allograft and its life, should receive if necessary psychological counseling.

Our first case of successful conception should not encourage other recipients of child bearing age to have a gestation, we must to inform to what they are facing, and in case of deciding on gestation prepare it for this in the best clinical conditions and plan the same.

\section{References}

1. Zetina-Tun HJ. Chapter 3. Selection and management of the candidate for heart transplantation. In: Careaga-Reyna G, ZetinaTun HJ. Organosocratic transplant in Mexico. 1st. Ed.The Modern Manual. 2014. Mexico, pp. 23-33.

2. Brosens I, Brosens J, Giuseppe Benagiano. The risk of obstetrical syndromes after solid organ transplantation. Best Practice \& Research Clinical Obstetrics and Gynecology. 2014;28(8):12111221.

3. Wu D, Wilt J, Restaino S. Pregnancy after thoracic organ transplantation. Sem Perinatol. 2007;31(6):354-362.

4. McKay DB, Josephson MA, Armenti VT, August P, Coscia LA, Davis $\mathrm{CL}$, et al. Reproduction and transplantation: report on the AST Consensus Conference on Reproductive Issues and Transplant. Am J Transplant. 2005;5(7):1592-1599.

5. Armenia V, Constantinescu S, Moritz M. Pregnancy after transplantation. Transplant Rev 2008;22:223-240.

6. Kim K, Sukhani R, Slogoff S, Tomich PG. Central hemodynamic changes associated with pregnancy in long-term cardiac transplant recipient. Am J Obstet Gynecol. 1996;174(5):1651-1653.

7. Miniero R, Tardío I, P Centofanti, C Goggi, C Mammana, F Parisi, et al. Pregnancy in heart transplant recipients. J Heart Lung Transplant. 2004;23(7):898-901.
8. Durst J, Rampersad R. Pregnancy in women with solid-organ transplants: A review. Obstet and Gynecol Surv. 2015;70(6):408418. doi: 10.1097/OGX.0000000000000194

9. VosR,RuttensD,VerledenSE,VandermeulenE,BellonH,Vanaudenaerde $\mathrm{BM}$, etal. Pregnancy after heartand lung transplantation. Best Practice \& Research Clinical Obstetrics and Gynecology. 2014;28(8):11461162. doi: 10.1016/j.bpobgyn.2014.07.019

10. Cowan S, Davison J, Doria C, Moritz MJ, Armenti VT. Pregnancy after cardiac transplantation. Cardiol Clin. 2012;30(3):441-452. doi: 10.1016/j.ccl.2012.05.001

11. Coscia LA, Constantinescu S, Moritz MJ, FrankAM, Ramirez CB,Maley WR, et al. Report from the National Transplantation Pregnancy Registry (NTPR): outcomes of pregnancy after transplantation. Clin Transpl. 2010:65-85.

12. Mc Kay DB, Josephson MA. Pregnancy in recipients of solid organseffects on mother and child. N Engl J Med. 2006;354:1281-1293. DOI: 10.1056/NEJMra050431

13. Bhagra C, Bhagra S, Donado A, But T, Forrest L, MacGowan G, et al. Pregnancy in cardiac transplant recipients. Clin Transplant. 2016;30(9):1059-1065. doi: 10.1111/ctr.12788

14. Thakrar M, Morley K, Lordan J, Meachery G, Fisher AJ, Parry G, et al. Pregnancy after lung and heart lung transplantation. J Heart Lung Transplant. 2014;33(6):593-598. doi: 10.1016/j.healun.2014.02.008

15. Abdalla A, Mancini D. Management of pregnancy in the postcardiac transplant patient. Sem Perinatol. 2014;38(5):318-325. doi: 10.1053/j.semperi.2014.04.022

16. Fuchs KM, Coustan DR. Immunosuppressant therapy in pregnant organ transplant recipients. Semin Perinatol. 2007;31(6):363-371.

17. O’Boyle P, Smith J, Danskine A, Lyster S, Burke MM, Banner NR. Denovo HLA sensitization and antibody mediated rejection following pregnancy in a heart transplant recipient. Am J Transplant. 2010;10(1):180-183. doi: 10.1111/j.1600-6143.2009.02875.x

18. Davison JM. Dialysis, transplantation and pregnancy. Am J Kidney Dis. 1991;17(2):127-132.

19. Lou RJ, Scott JR. Pregnancy following renal transplantation. Clin Obstet Gynecol. 1985;28(2):339-350. 